\title{
AN INTEGRO-DIFFERENTIAL EQUATION ARISING FROM AN ELECTROCHEMISTRY MODEL
}

\author{
BY \\ Y. S. CHOI (Department of Mathematics, University of Connecticut, Storrs) \\ AND
}

ROGER LUI (Dept. of Mathematical Sciences, Worcester Polytechnic Institute, Worcester, MA)

\begin{abstract}
In this paper, we prove the existence and uniqueness of steady-state solutions for a system of equations arising from a model in electrochemistry. The same result was established by the authors in an earlier paper under the additional assumptions that the space-dimension $N=2$ and the concentrations of the charged ions satisfy an electro-neutrality condition.
\end{abstract}

1. Introduction. Let $\Omega^{*}, \omega_{1}, \omega_{2}$ be open, bounded, connected subsets of $\mathbf{R}^{N}, N \geq 2$ such that $\overline{\omega_{1}}, \overline{\omega_{2}} \subset \Omega^{*}$ and $\overline{\omega_{1}} \cap \overline{\omega_{2}}=\varnothing$. Let $\Gamma_{1}, \Gamma_{2}, \Gamma_{3}$ be the $C^{2+\gamma}$ boundaries of $\omega_{1}, \omega_{2}$ and $\Omega^{*}$, respectively, where $0<\gamma<1$ and define $\Omega=\Omega^{*} \backslash\left(\overline{\omega_{1}} \cup \overline{\omega_{2}}\right)$. The purpose of this paper is to prove the following theorem:

TheOREM 1.1. Let $\Omega$ be defined as above and let $\varepsilon, C_{i}$ be positive and $z_{i}$ be nonzero constants for $i=1, \ldots, m$. Suppose $Q \in C^{\gamma}(\bar{\Omega})$ where $0<\gamma<1$. Then the equation

$$
\varepsilon \Delta \phi=-\sum \frac{z_{i} C_{i} e^{-z_{i} \phi(x)}}{\int_{\Omega} e^{-z_{i} \phi(y)} d y}+Q(x)
$$

subject to the boundary conditions

$$
\left\{\begin{aligned}
\phi(x) & =0 \text { for } x \in \Gamma_{1}, \quad \phi(x)=\alpha \text { for } x \in \Gamma_{2}, \\
\frac{\partial \phi}{\partial n}(x) & =0 \text { for } x \in \Gamma_{3}
\end{aligned}\right.
$$

have a unique solution $\phi \in C^{2+\gamma}(\bar{\Omega})$.

The above equations arise from the study of an electrochemistry model [1], [2], [3]. Consider a container holding some electrolyte, which is a mixture of charged particles in a solution. Two electrodes are inserted into the electrolyte and are subject to prescribed electric potentials. The densities of the charged particles are denoted by $u_{1}, \ldots, u_{m}$ and

Received November 18, 1994.

1991 Mathematics Subject Classification. Primary 45K05.

The first author's research was partially supported by NSF grant no. DMS-9208636. Fax: 203-486-4238.

The second author's research was partially supported by NSF grant no. DMS-9202341. 
the electric potential is denoted by $\phi$. Each species has a characteristic mobility constant $\Omega_{i}$ such that its velocity under the influence of the electric field is given by $-\Omega_{i} z_{i} \nabla \phi$ where $z_{i}$ is its charge. The charge $z_{i}$ must be integer-valued, and may be positive, negative, or zero. The species also diffuse with diffusion constant $d_{i}>0$. It is frequently assumed that $\Omega_{i}=\mu d_{i}$ for $i=1, \ldots, m$ where $\mu>0$. This is called the Einstein relation. Under the above assumptions, the flux of species $i$ is given by $-d_{i}\left(\nabla u_{i}+\mu z_{i} u_{i} \nabla \phi\right)$ and, after non-dimensionalization, $u_{i}$ and $\phi$ satisfy the following system of equations:

$$
\left\{\begin{aligned}
\frac{\partial u_{i}}{\partial t} & =d_{i} \operatorname{div}\left(\nabla u_{i}+\mu z_{i} u_{i} \nabla \phi\right), \quad i=1, \ldots, m, \\
\varepsilon \Delta \phi & =-e \sum z_{k} u_{k}+Q(x) .
\end{aligned}\right.
$$

In the above equations, $\varepsilon>0$ is the permittivity of the solvent, $e>0$ is the molar charge, and $Q$ may be thought of as a charge density distributed inside the container.

Equations (1.3) are solved subject to the following boundary conditions:

$$
\left\{\begin{array}{l}
\left(\nabla u_{i}+\mu z_{i} u_{i} \nabla \phi\right) \cdot \mathbf{n}=0, \quad \text { for } x \in \partial \Omega, t>0 \\
\phi(x, t)=0 \quad \text { for } x \in \Gamma_{1}, \quad \phi(x, t)=\alpha \quad \text { for } x \in \Gamma_{2}, t>0 \\
\frac{\partial \phi}{\partial n}(x, t)=0 \quad \text { for } x \in \Gamma_{3}, t>0
\end{array}\right.
$$

and initial conditions

$$
u_{i}(x, 0)=u_{i}^{0}(x) \geq 0, \quad i=1, \ldots, m .
$$

In (1.4), $\partial \Omega=\Gamma_{1} \cup \Gamma_{2} \cup \Gamma_{3}, \mathbf{n}$ is the unit outward normal at $\partial \Omega$, and $\partial \phi / \partial n=\nabla \phi \cdot \mathbf{n}$. Without loss of generality, we assume that $\alpha$ in (1.4) is positive.

The initial condition $\phi(x, 0)=\phi^{0}(x)$ is not prescribed because it can be obtained from solving the equation $\varepsilon \Delta \phi^{0}=-e \sum z_{k} u_{k}^{0}+Q(x)$ together with the boundary conditions $\left.\phi^{0}\right|_{\Gamma_{1}}=0,\left.\phi^{0}\right|_{\Gamma_{2}}=\alpha, \partial \phi^{0} /\left.\partial n\right|_{\Gamma_{3}}=0$. It is obvious that by absorbing $\mu$ into $\phi$ and $e$ into $\varepsilon$, we may assume that $\mu=e=1$. We also assume that $z_{i} \neq 0$ since if $z_{i}=0$, then $u_{i}$ is decoupled from the system (1.3) and may be solved independently.

The evolution problem (1.3), (1.4) has been studied in [3] with $Q(x) \equiv 0$. Using the techniques developed in [3], one can show that given $T>0$, there exist unique, smooth solutions $u_{i}(x, t), i=1, \ldots, m$ and $\phi(x, t)$ to equations (1.3), (1.4), and (1.5) for $0 \leq t<T$. Furthermore, $u_{i}>0$ if $t>0$. Also, from (1.3), and (1.4a), $\int_{\Omega} u_{i}(x, t) d x=C_{i}$ is independent of $t$ and depends only on the initial data $u_{i}^{0}$.

The steady-state solutions $u_{i}(x), i=1, \ldots, m$ and $\phi(x)$ satisfy the equations

$$
\left\{\begin{aligned}
\operatorname{div}\left(\nabla u_{i}+z_{i} u_{i} \nabla \phi\right) & =0, \quad i=1, \ldots, m, \\
\varepsilon \Delta \phi & =-\sum z_{k} u_{k}+Q(x), \\
\int_{\Omega} u_{i}(x) d x=C_{i}>0, \quad i=1, \ldots, m & \text { for } x \in \Gamma_{1}, \quad \phi(x)=\alpha \text { for } x \in \Gamma_{2}, \\
\phi(x)=0 \text { for } x \in \Gamma_{3} &
\end{aligned}\right.
$$

where $C_{i}, i=1, \ldots, m$ are known constants. Let $v_{i}=u_{i} e^{z_{i} \phi}$ for $i=1, \ldots, m$. Then $v_{i}$ satisfies the equation $\operatorname{div}\left(e^{-z_{i} \phi} \nabla v_{i}\right)=0$ and the boundary conditions $\partial v_{i} /\left.\partial n\right|_{\partial \Omega}=0$. 
Multiplying this equation by $v_{i}$ and integrating by parts, we see that $v_{i}$ is a constant. This constant may be found from condition (1.6c). Doing so, we obtain

$$
u_{i}(x)=\frac{C_{i} e^{-z_{i} \phi(x)}}{\int_{\Omega} e^{-z_{i} \phi(y)} d y} \quad \text { for } i=1, \ldots, m .
$$

Substituting this into Eq. (1.6b), we obtain Eq. (1.1). It is clear from above that proving the existence and uniqueness of solutions to Eqs. (1.6) is equivalent to proving the existence and uniqueness of solutions to Eq. (1.1) subject to the boundary conditions (1.2).

In the paper [3], existence and uniqueness of steady-state solutions to Eqs. (1.6) were established under the assumption $Q \equiv 0$ and the electro-neutrality condition $\sum z_{i} C_{i}=0$. In the same paper, global stability of the steady-state solutions was also established when $N=2$. This result is equivalent to saying that ions in any initial distribution will eventually settle down to their unique steady-states. Due to the lack of time-independent a priori bounds for $\left\|u_{i}(\cdot, t)\right\|_{L^{2}(\Omega)}$, the global stability result cannot be extended to the case $N=3$.

The purpose of this paper is to prove the existence and uniqueness of steady-state solutions to Eqs. (1.6) without the assumption $Q \equiv 0$ or $\sum z_{i} C_{i}=0$. The result is stated in Theorem 1.1. As a corollary of this theorem and Theorem 8.1 of [3], we have the following stability result.

Theorem 1.2. Let $u_{i}^{0} \geq 0$ be smooth initial data defined on $\Omega \subset \mathbf{R}^{2}$ for $i=1, \ldots, m$ and let $C_{i}=\int_{0}^{1} u_{i}^{0}(x) d x>0$. For $N=2$, there exist unique solutions $U_{i}, i=1, \ldots, m$ and $\Phi$ to the steady-state equations (1.6). Moreover, the solutions $u_{i}, i=1, \ldots, m$ and $\phi$ of equations (1.3), (1.4), and (1.5) satisfy $\lim _{t \rightarrow \infty} u_{i}(x, t)=U_{i}(x)$ and $\lim _{t \rightarrow \infty} \phi(x, t)=$ $\Phi(x)$ uniformly on $\bar{\Omega}$.

The same result holds for $\Omega \subset \mathbf{R}^{3}$ provided that we have a time-dependent $L^{2}(\Omega)$ norm bound of the solutions $u_{i}, i=1, \ldots, m$.

The organization of this paper is as follows. Section 2 contains the proof of a priori bounds for the steady-state solutions while Sec. 3 contains the proof of Theorem 1.1.

2. A priori estimates. Let $\Omega_{+}=\{x \in \bar{\Omega} \mid u \geq 0\}$ and $\Omega_{-}=\{x \in \bar{\Omega} \mid u<0\}$. We first establish two lemmas.

LEMMA 2.1. Let $w, u \in C(\bar{\Omega})$, and $w \geq 0$ on $\bar{\Omega}$. Then for any nonnegative integer $n$

$$
z_{i} \frac{\int_{\Omega_{+}} w(x) e^{-z_{i} u} u^{n+1} d x}{\int_{\Omega_{+}} w(x) e^{-z_{i} u} u^{n} d x} \leq z_{i} \frac{\int_{\Omega_{+}} w(x) u^{n+1} d x}{\int_{\Omega_{+}} w(x) u^{n} d x}, \quad \text { if } z_{i}>0 .
$$

Proof. Define positive measures $M_{i}$ and $m_{i}$ on $\Omega_{+}$such that their Radon-Nikodym derivatives satisfy $d M_{i} / d x=e^{-z_{i} u} w(x) u^{n} / \int_{\Omega_{+}} e^{-z_{i} u} w(x) u^{n} d x$ and $d m_{i} / d x=$ $w(x) u^{n} / \int_{\Omega_{+}} w(x) u^{n} d x$, respectively. Then, $\int_{\Omega_{+}} d M_{i}=1$ and $\int_{\Omega_{+}} d m_{i}=1$. 
Suppose $z_{i}>0$. From Jensen's inequality

$$
\begin{aligned}
\exp \left(\frac{z_{i} \int_{\Omega_{+}} w(x) e^{-z_{i} u} u^{n+1} d x}{\int_{\Omega_{+}} w(x) e^{-z_{i} u} u^{n} d x}\right) & =\exp \left(\int_{\Omega_{+}} z_{i} u d M_{i}\right) \\
& \leq \int_{\Omega_{+}} e^{z_{i} u} d M_{i} \\
& =\frac{\int_{\Omega_{+}} w(x) u^{n} d x}{\int_{\Omega_{+}} w(x) e^{-z_{i} u} u^{n} d x} \\
& =1 / \int_{\Omega_{+}} e^{-z_{i} u} d m_{i} .
\end{aligned}
$$

Taking the logarithm on both sides, we have

$$
\begin{aligned}
\frac{z_{i} \int_{\Omega_{+}} w(x) e^{-z_{i} u} u^{n+1} d x}{\int_{\Omega_{+}} w(x) e^{-z_{i} u} u^{n} d x} & \leq-\log \left(\int_{\Omega_{+}} e^{-z_{i} u} d m_{i}\right) \\
& \leq \int_{\Omega_{+}} z_{i} u d m_{i} \\
& =\frac{z_{i} \int_{\Omega_{+}} w(x) u^{n+1} d x}{\int_{\Omega_{+}} w(x) u^{n} d x} .
\end{aligned}
$$

The proof of the lemma is complete.

Lemma 2.2. Let $n$ be a nonnegative integer and let $\phi_{0}, u \in C(\bar{\Omega})$. Then

$$
z_{i} C_{i} \frac{\int_{\Omega} e^{-z_{i} \phi_{0}} e^{-z_{i} u} u^{2 n+1} d x}{\int_{\Omega} e^{-z_{i} \phi_{0}} e^{-z_{i} u} d x} \leq\left|z_{i}\right| C_{i} \frac{\int_{\Omega} e^{-z_{i} \phi_{0}}|u|^{2 n+1} d x}{\int_{\Omega} e^{-z_{i} \phi_{0}} d x} .
$$

Proof. Suppose $z_{i}>0$. From Eq. (2.1), we have

$$
\begin{aligned}
z_{i} C_{i} \frac{\int_{\Omega} e^{-z_{i} \phi_{0}} e^{-z_{i} u} u^{2 n+1} d x}{\int_{\Omega} e^{-z_{i} \phi_{0}} e^{-z_{i} u} d x} \\
\leq z_{i} C_{i} \frac{\int_{\Omega_{+}} e^{-z_{i} \phi_{0}} e^{-z_{i} u} u^{2 n+1} d x}{\int_{\Omega} e^{-z_{i} \phi_{0}} e^{-z_{i} u} d x} \\
\quad=z_{i} C_{i} \frac{\int_{\Omega_{+}} e^{-z_{i} \phi_{0}} e^{-z_{i} u} d x}{\int_{\Omega} e^{-z_{i} \phi_{0}} e^{-z_{i} u} d x} \frac{\int_{\Omega_{+}} e^{-z_{i} \phi_{0}} e^{-z_{i} u} u d x}{\int_{\Omega_{+}} e^{-z_{i} \phi_{0}} e^{-z_{i} u} d x} \cdots \frac{\int_{\Omega_{+}} e^{-z_{i} \phi_{0}} e^{-z_{i} u} u^{2 n+1} d x}{\int_{\Omega_{+}} e^{-z_{i} \phi_{0}} e^{-z_{i} u} u^{2 n} d x} \\
\leq z_{i} C_{i} \frac{\int_{\Omega_{+}} e^{-z_{i} \phi_{0}} e^{-z_{i} u} d x}{\int_{\Omega} e^{-z_{i} \phi_{0}} e^{-z_{i} u} d x} \frac{\int_{\Omega_{+}} e^{-z_{i} \phi_{0}} u d x}{\int_{\Omega_{+}} e^{-z_{i} \phi_{0}} d x} \cdots \frac{\int_{\Omega_{+}} e^{-z_{i} \phi_{0}} u^{2 n+1} d x}{\int_{\Omega_{+}} e^{-z_{i} \phi_{0}} u^{2 n} d x} \\
\quad=z_{i} C_{i} \frac{\int_{\Omega_{+}} e^{-z_{i} \phi_{0}} e^{-z_{i} u} d x}{\int_{\Omega} e^{-z_{i} \phi_{0}} e^{-z_{i} u} d x} \frac{\int_{\Omega_{+}} e^{-z_{i} \phi_{0}} u^{2 n+1} d x}{\int_{\Omega_{+}} e^{-z_{i} \phi_{0}} d x} \\
\leq z_{i} C_{i} \frac{\int_{\Omega_{+}} e^{-z_{i} \phi_{0}} u^{2 n+1} d x}{\int_{\Omega} e^{-z_{i} \phi_{0}} d x} \\
\leq\left|z_{i}\right| C_{i} \frac{\int_{\Omega} e^{-z_{i} \phi_{0}}|u|^{2 n+1} d x}{\int_{\Omega} e^{-z_{i} \phi_{0}} d x} .
\end{aligned}
$$


The second to last of the above inequalities follows from the fact that

$$
\begin{aligned}
& \left(\int_{\Omega_{+}} e^{-z_{i} \phi_{0}} e^{-z_{i} u} d x\right)\left(\int_{\Omega_{+}} e^{-z_{i} \phi_{0}} d x+\int_{\Omega_{-}} e^{-z_{i} \phi_{0}} d x\right) \\
& \quad \leq\left(\int_{\Omega_{+}} e^{-z_{i} \phi_{0}} e^{-z_{i} u} d x+\int_{\Omega_{-}} e^{-z_{i} \phi_{0}} e^{-z_{i} u} d x\right)\left(\int_{\Omega_{+}} e^{-z_{i} \phi_{0}} d x\right) .
\end{aligned}
$$

Hence, inequality (2.3) is valid for $z_{i}>0$.

If $z_{i}<0$, let $v=z_{i} u$. From (2.3), with $z_{i}=1$ and $\phi_{0}$ there replaced by $z_{i} \phi_{0}$,

$$
\begin{aligned}
z_{i} C_{i} & \frac{\int_{\Omega} e^{-z_{i} \phi_{0}} e^{-z_{i} u} u^{2 n+1} d x}{\int_{\Omega} e^{-z_{i} \phi_{0}} e^{-z_{i} u} d x} \\
& =\frac{C_{i}}{z_{i}^{2 n}} \frac{\int_{\Omega} e^{-z_{i} \phi_{0}} e^{-v} v^{2 n+1} d x}{\int_{\Omega} e^{-z_{i} \phi_{0}} e^{-v} d x} \\
& \leq \frac{C_{i}}{z_{i}^{2 n}} \frac{\int_{\Omega} e^{-z_{i} \phi_{0}}|v|^{2 n+1} d x}{\int_{\Omega} e^{-z_{i} \phi_{0}} d x} \\
& =\left|z_{i}\right| C_{i} \frac{\int_{\Omega} e^{-z_{i} \phi_{0}}|u|^{2 n+1} d x}{\int_{\Omega} e^{-z_{i} \phi_{0}} d x} .
\end{aligned}
$$

The proof of the lemma is complete.

We now define $\phi_{0} \in C^{2+\gamma}(\bar{\Omega})$ as the solution to the equation

$$
\varepsilon \Delta \phi_{0}=Q(x)
$$

subject to the boundary conditions (1.2). Let $u=\phi-\phi_{0}$. Then $u$ satisfies

$$
\varepsilon \Delta u=-\sum \frac{z_{i} C_{i} e^{-z_{i} \phi_{0}} e^{-z_{i} u}}{\int_{\Omega} e^{-z_{i} \phi_{0}(y)} e^{-z_{i} u(y)} d y}
$$

and the homogeneous boundary conditions

$$
\left\{\begin{aligned}
u(x)=0 & \text { for } x \in \Gamma_{1}, \quad u(x)=0 \quad \text { for } x \in \Gamma_{2}, \\
\frac{\partial u}{\partial n}(x)=0 & \text { for } x \in \Gamma_{3} .
\end{aligned}\right.
$$

Proving Theorem 1.1 is equivalent to showing that Eqs. (2.5) and (2.6) have a unique solution in $C^{2+\gamma}(\bar{\Omega})$.

In what follows, $k, k_{1}, k_{2}, \ldots$, and $M, M_{1}, M_{2}, \ldots$ will denote generic positive constants. From the definition of $\phi_{0}$, we have $\left\|\phi_{0}\right\|_{\infty} \leq k_{1}$.

Let $\bar{z}=\max \left|z_{i}\right|$ and $|\Omega|=\operatorname{meas}(\Omega)$. Multiplying Eq. (2.5) by $-u^{2 n+1}$ and integrating over $\Omega$, we have, from $(2.3)$,

$$
\begin{aligned}
\varepsilon \frac{2 n+1}{(n+1)^{2}} \int_{\Omega} \nabla\left(u^{n+1}\right) \cdot \nabla\left(u^{n+1}\right) d x & =\sum z_{i} C_{i} \frac{\int_{\Omega} e^{-z_{i} \phi_{0}} e^{-z_{i} u} u^{2 n+1} d x}{\int_{\Omega} e^{-z_{i} \phi_{0}} e^{-z_{i} u} d y} \\
& \leq \sum\left|z_{i}\right| C_{i} \frac{\int_{\Omega} e^{-z_{i} \phi_{0}}|u|^{2 n+1} d x}{\int_{\Omega} e^{-z_{i} \phi_{0}} d y} \\
& \leq M \int_{\Omega}|u|^{2 n+1} d x
\end{aligned}
$$


where $M=\sum\left|z_{i}\right| C_{i} e^{2 \bar{z} k_{1}} /|\Omega|$. In particular, taking $n=0$ in Eq. (2.7) and employing Poincaré's inequality, we have

$$
\|u\|_{H^{1}}^{2} \leq \frac{M}{\varepsilon}\|u\|_{1} \leq \frac{M}{\varepsilon} k_{2}\|u\|_{2} \leq \frac{M}{\varepsilon} k_{3}\|u\|_{H^{1}} .
$$

Thus $\|u\|_{H^{1}} \leq k_{4}$ and we conclude that

$$
\|u\|_{2} \leq k_{5}
$$

We now bootstrap this estimate to obtain an $L^{\infty}$ bound on $u$. We concentrate on the case $N \geq 3$ since the case $N=2$ is easier.

Lemma 2.3. Let $N \geq 3$ and let $n \geq 1$ be a positive integer. Define

$$
p=2 N(n+1) /(N-2) \text {. }
$$

Then there exists a constant $M_{1}$, independent of $n$, such that

$$
\|u\|_{p} \leq M_{1} p^{(N+1) /(N+4)} .
$$

Proof. For any $w \in H^{1}(\Omega)$ with $w=0$ on part of the boundary $\partial \Omega$, Sobolev's inequality implies that

$$
\|w\|_{2 N /(N-2)} \leq k\|w\|_{H^{1}}
$$

From (2.7),

$$
\|u\|_{2 N(n+1) /(N-2)}^{2 n+2}=\left\|u^{n+1}\right\|_{2 N /(N-2)}^{2} \leq k^{2}\left\|u^{n+1}\right\|_{H^{1}}^{2} \leq \frac{k^{2} M}{\varepsilon} \frac{(n+1)^{2}}{(2 n+1)}\|u\|_{2 n+1}^{2 n+1} .
$$

Now we employ the interpolation equation (7.9) on p. 146 of [4] to obtain

$$
\|u\|_{2 N(n+1) /(N-2)}^{2 n+2} \leq \frac{k^{2} M}{\varepsilon} \frac{(n+1)^{2}}{(2 n+1)}\left(\|u\|_{2}^{\lambda}\|u\|_{2 N(n+1) /(N-2)}^{1-\lambda}\right)^{2 n+1}
$$

where $\lambda$ satisfies

$$
\frac{1}{2 n+1}=\frac{\lambda}{2}+\frac{1-\lambda}{2 N(n+1) /(N-2)} .
$$

It can be checked that

$$
\begin{gathered}
\lambda=\frac{N+4 n+2}{(2 n+1)(n N+2)}, \\
1+\lambda(2 n+1)=\frac{(n+1)(N+4)}{n N+2},
\end{gathered}
$$

and

$$
\frac{1}{1+\lambda(2 n+1)} \rightarrow \frac{N}{N+4} \quad \text { as } n \rightarrow \infty
$$

From (2.11),

$$
\|u\|_{2 N(n+1) /(N-2)}^{1+\lambda(2 n+1)} \leq \frac{k^{2} M}{\varepsilon} \frac{(n+1)^{2}}{(2 n+1)}\|u\|_{2}^{(2 n+1) \lambda} \leq \frac{2 k^{2} n M}{\varepsilon}\|u\|_{2}^{(2 n+1) \lambda} .
$$

From (2.8),

$$
\|u\|_{2 N(n+1) /(N-2)} \leq k_{1} n^{1 /(1+\lambda(2 n+1))}
$$


where $k_{1} \geq 1$ is independent of $n$. From (2.14),

$$
\|u\|_{2 N(n+1) /(N-2)} \leq k_{1} n^{(N+1) /(N+4)}
$$

for sufficiently large $n$. By increasing $k_{1}$ if necessary, the above inequality holds for all n. From (2.9),

$$
\|u\|_{p} \leq k_{1}\left(\frac{p(N-2)}{2 N}-1\right)^{(N+1) /(N+4)} \leq M_{1} p^{(N+1) /(N+4)} .
$$

The proof of the lemma is complete.

Corollary 2.4. For any $1 \leq p<\infty$,

$$
\|u\|_{p}<\infty
$$

LEMmA 2.5. Let $N \geq 3$. For any integer $r \geq 4 N /(N-2)$, there exists $M_{2}>0$, independent of $r$, such that

$$
\|u\|_{r} \leq M_{2} r^{(N+1) /(N+4)} .
$$

Proof. Given $r \geq 4 N /(N-2)$, choose an integer $s$ such that

$$
\frac{2 N s}{N-2}<r \leq \frac{2 N(s+1)}{N-2}
$$

Define $p=2 N(s+1) /(N-2)$. Then it is easy to see that $0 \leq 1 / r-1 / p<1$. Hence $|\Omega|^{1 / r-1 / p} \leq k_{1}$. From Hölder's inequality,

$$
\|u\|_{r} \leq|\Omega|^{1 / r-1 / p}\|u\|_{p}
$$

From Lemma 2.3,

$$
\begin{aligned}
\|u\|_{r} & \leq k_{1} M_{1} p^{(N+1) /(N+4)} \\
& \leq k_{1} M_{1}\left(\frac{4 N s}{N-2}\right)^{(N+1) /(N+4)} \\
& \leq M_{2} r^{(N+1) /(N+4)} .
\end{aligned}
$$

This completes the proof of the lemma.

LEMma 2.6. For any constant $\beta \in \mathbf{R}$, there exists $M_{\beta}>0$ such that

$$
\int_{\Omega} e^{\beta u} d x \leq M_{\beta}
$$

Proof. Since

$$
\left|\int_{\Omega} e^{\beta u} d x\right| \leq \sum_{r=0}^{\infty} \int_{\Omega} \frac{(|\beta u|)^{4}}{r !} d x
$$

and $u \in L^{r}$ for any positive integer $r$ because of Corollary 2.4, it suffices to show that the above series converges.

From (2.15), we have, for any $r_{0}>4 N /(N-2)$

$$
\sum_{r=r_{0}}^{\infty} \int_{\Omega} \frac{|\beta u|^{r}}{r !} d x \leq \sum_{r=r_{0}}^{\infty} \frac{|\beta|^{r}}{r !}\|u\|_{r}^{r} \leq \sum_{r=r_{0}}^{\infty} \frac{|\beta|^{r}}{r !} M_{2}^{r} r^{(N+1) r /(N+4)}
$$


Stirling's formula implies that

$$
\lim _{r \rightarrow \infty} \frac{r^{r} \sqrt{2 \pi r}}{e^{r} r !}=1 .
$$

Thus by increasing $r_{0}$ if necessary, for $r \geq r_{0}$,

$$
\frac{1}{r !} \leq \frac{2 e^{r}}{r^{r} \sqrt{2 \pi r}}
$$

Therefore,

$$
\sum_{r=r_{0}}^{\infty} \int_{\Omega} \frac{|\beta u|^{r}}{r !} d x \leq \sum_{r=r_{0}}^{\infty} \frac{2 e^{r} M_{2}^{r}|\beta|^{r}}{r^{r} \sqrt{2 \pi r}} r^{(N+1) r /(N+4)}=\sum_{r=r_{0}}^{\infty} \frac{2\left(e M_{2}|\beta|\right)^{r}}{r^{3 r /(N+4)} \sqrt{2 \pi r}} .
$$

This series converges because of the root test and (2.17) follows. The lemma is also valid if $N=2$ since in this case there exist constants $c_{1}, c_{2}>0$ such that $\int_{\Omega} \exp \left(c_{1}|u| /\|u\|_{H^{1}}\right)^{2} d x$ $\leq c_{2}$ (see p. 162 in [4]) and $\|u\|_{H^{1}}$ has been shown to be bounded.

Lemma 2.7. For any $1 \leq p<\infty$, there exists an $M_{p}>0$ such that

$$
\frac{1}{\int_{\Omega} e^{-z_{i} \phi_{0}(y)} e^{-z_{i} u(y)} d y}\left\|e^{-z_{i} \phi_{0}} e^{-z_{i} u}\right\|_{p} \leq M_{p} .
$$

Proof. Recall that $\left\|\phi_{0}\right\|_{\infty} \leq k_{1}$ and $\bar{z}=\max \left|z_{i}\right|$. From Lemma 2.6,

$$
\left\|e^{-z_{i} u}\right\|_{p} \leq k_{2}
$$

where $k_{2}$ may depend on $p$. Hence

$$
\left\|e^{-z_{i} \phi_{0}} e^{-z_{i} u}\right\|_{p} \leq k_{2} e^{\bar{z} k_{1}} .
$$

The function $f(v)=1 / v$ is convex so that Jensen's inequality implies that

$$
\left(\frac{1}{|\Omega|} \int_{\Omega} e^{-z_{i} u(y)} d y\right)^{-1} \leq \frac{1}{|\Omega|} \int_{\Omega} e^{z_{i} u(y)} d y
$$

Since

$$
\int_{\Omega} e^{-z_{i} \phi_{0}(y)} e^{-z_{i} u(y)} d y \geq e^{-\bar{z} k_{1}} \int_{\Omega} e^{-z_{i} u(y)} d y
$$

we have

$$
\frac{1}{\int_{\Omega} e^{-z_{i} \phi_{0}(y)} e^{-z_{i} u(y)} d y} \leq \frac{e^{\bar{z} k_{1}}}{|\Omega|^{2}} \int_{\Omega} e^{z_{i} u(y)} d y \leq k_{3} .
$$

Inequality (2.21) follows from (2.23) and (2.26).

LEMmA 2.8. There exists an $M_{3}>0$ such that

$$
\|u\|_{C^{2+\gamma}(\bar{\Omega})} \leq M_{3} .
$$

Proof. From Lemma 2.7, the right-hand side of $(2.5)$ is bounded in the $L^{p}$ norm for any $1 \leq p<\infty$. Thus $u \in W^{2, p}$ by regularity estimates. By taking $p$ sufficiently large, Sobolev's imbedding theorem implies that $u \in C^{1+\gamma}(\bar{\Omega})$. Since $\phi_{0} \in C^{2+\gamma}(\bar{\Omega})$, the righthand side of Eq. $(2.5)$ is bounded in the $C^{1+\gamma}(\bar{\Omega})$ norm. Schauder's estimate then gives a bound in the $C^{2+\gamma}(\bar{\Omega})$ norm. The proof of the lemma is complete. 
REMARK. Suppose the right-hand side of Eq. (2.5) is multiplied by $\lambda \in[0,1]$ and that the same a priori bounds (2.27) for this new equation subject to the same boundary condition (2.6) also hold. In other words, $M_{3}$ can be taken to be independent of $\lambda \in[0,1]$. This is important in our proof in the next section.

3. Proof of Theorem 1.1. Let $M_{3}$ be defined as in Lemma 2.8 and let

$$
S=\left\{u \in C^{\gamma}(\bar{\Omega}): u=0 \text { on } \Gamma_{1} \cup \Gamma_{2}, \partial u / \partial n=0 \text { on } \Gamma_{3},\|u\|_{C^{\gamma}(\bar{\Omega})} \leq M_{3}\right\} .
$$

We employ the notation $\Delta^{-1}(f)$ to denote the solution $w$ of $\Delta w=f$ satisfying the boundary conditions (2.6).

Consider the map $T_{\lambda}: S \rightarrow C^{\gamma}(\bar{\Omega})$ such that for any $u \in S$,

$$
T_{\lambda}(u)=u+\Delta^{-1}\left(\lambda \sum \frac{z_{i} C_{i} e^{-z_{i} \phi_{0}} e^{-z_{i} u}}{\int_{\Omega} e^{-z_{i} \phi_{0}(y)} e^{-z_{i} u(y) d y}}\right) .
$$

From Lemma 2.8 and its following remark, there is no solution to $T_{\lambda}(u)=0$ on $\partial S$ for any $0 \leq \lambda \leq 1$. Thus $\operatorname{deg}\left(T_{\lambda}, S, 0\right)$ is well-defined for $0 \leq \lambda \leq 1$. By the homotopy property, $\operatorname{deg}\left(T_{0}, S, 0\right)=\operatorname{deg}\left(T_{1}, S, 0\right)$. Since $T_{0}$ is the identity $\operatorname{map}, \operatorname{deg}\left(T_{0}, S, 0\right)=1$. Therefore $\operatorname{deg}\left(T_{1}, S, 0\right)=1$ and a solution of the equation $T_{1}(u)=0$ exists. By the regularity estimate, $u \in C^{2+\gamma}(\bar{\Omega})$. With $\phi=\phi_{0}+u$ and $\phi_{0} \in C^{2+\gamma}(\bar{\Omega})$, we have $\phi \in C^{2+\gamma}(\bar{\Omega})$. This proves the existence of a solution to Eqs. (1.1)-(1.2).

Next define

$$
U=\left\{u \in C^{1}(\bar{\Omega}): u=0 \text { on } \Gamma_{1} \cup \Gamma_{2}, \partial u / \partial n=0 \text { on } \Gamma_{3}\right\},
$$

and $I: U \rightarrow \mathbf{R}$ by

$$
I(u)=\int_{\Omega} \frac{\varepsilon}{2}|\nabla u|^{2} d x+\sum C_{i} \log \int_{\Omega} e^{-z_{i} \phi_{0}} e^{-z_{i} u} d x .
$$

It can be checked that $I$ is Fréchet differentiable, and for any $u, v \in U$,

$$
I^{\prime}(u) v=\int_{\Omega} \varepsilon \nabla u \cdot \nabla v d x-\sum z_{i} C_{i} \frac{\int_{\Omega} e^{-z_{i} \phi_{0}} e^{-z_{i} u} v d x}{\int_{\Omega} e^{-z_{i} \phi_{0}} e^{-z_{i} u} d y} .
$$

In fact, $I \in C^{2}$. For any $u, v, w \in U$,

$$
\begin{aligned}
I^{\prime \prime}(u)(v, w)=\int_{\Omega} \varepsilon & \nabla w \cdot \nabla v d x+\sum z_{i}^{2} C_{i} \frac{\int_{\Omega} e^{-z_{i} \phi_{0}} e^{-z_{i} u} w v d x}{\int_{\Omega} e^{-z_{i} \phi_{0}} e^{-z_{i} u} d y} \\
& -\sum z_{i}^{2} C_{i} \frac{\int_{\Omega} e^{-z_{i} \phi_{0}} e^{-z_{i} u} v d x \int_{\Omega} e^{-z_{i} \phi_{0}} e^{-z_{i} u} w d x}{\left(\int_{\Omega} e^{-z_{i} \phi_{0}} e^{-z_{i} u} d y\right)^{2}} .
\end{aligned}
$$

Cauchy-Schwarz's inequality implies that

$$
\int_{\Omega} e^{-z_{i} \phi_{0}} e^{-z_{i} u} d x \int_{\Omega} e^{-z_{i} \phi_{0}} e^{-z_{i} u} v^{2} d x \geq\left(\int_{\Omega} e^{-z_{i} \phi_{0}} e^{-z_{i} u} v d x\right)^{2} .
$$

Let $v=w$ in Eq. (3.6). Then (3.7) implies that

$$
I^{\prime \prime}(u)(v, v) \geq \varepsilon \int_{\Omega}|\nabla v|^{2} d x \geq 0
$$


and

$$
I^{\prime \prime}(u)(v, v)=0 \quad \text { if and only if } \quad v=0 .
$$

We are now in a position to prove the uniqueness result in Theorem 1.1. Let $u_{1}$ and $u_{2}$ be two solutions of Eqs. (2.5)-(2.6). We can multiply Eq. (2.5) by any $v \in U$, and integrate by parts to obtain

$$
I^{\prime}\left(u_{i}\right) v=0 \text { for } i=1,2 \text {. }
$$

Now

$$
I^{\prime}\left(u_{2}\right) v-I^{\prime}\left(u_{1}\right) v=\int_{0}^{1} I^{\prime \prime}\left(u_{1}+t\left(u_{2}-u_{1}\right)\right)\left(u_{2}-u_{1}, v\right) d t
$$

Take $v=u_{2}-u_{1}$. Employing Eqs. (3.8) and (3.10), we have

$$
0=\int_{0}^{1} I^{\prime \prime}\left(u_{1}+t v\right)(v, v) d t \geq 0 .
$$

Since $I \in C^{2}$, so $I^{\prime \prime}\left(u_{1}+t v\right)(v, v)=0$ for all $t \in[0,1]$. Hence $v=u_{2}-u_{1}=0$ by Eq. (3.9). Thus, the solution $u$ to Eqs. $(2.5)-(2.6)$ is unique. The proof of Theorem 1.1 is complete.

Remark. For $N=2$, we can define

$$
V=\left\{u \in H^{1}(\Omega): u=0 \text { on } \Gamma_{1} \cup \Gamma_{2}, \partial u / \partial n=0 \text { on } \Gamma_{3}\right\},
$$

and $I: V \rightarrow \mathbf{R}$ by Eq. (3.4). Then we can proceed using variational calculus to show the existence and uniqueness of the solution to Eqs. (2.5)-(2.6). This approach fails for $N \geq 3$ since $e^{-z_{i} u}$ may not be integrable for $u \in V$. Moreover, the Fréchet derivative of $I$ may not exist, making it difficult to relate the critical points of $I$ to the weak solutions of Eqs. (2.5)-(2.6).

\section{REFERENCES}

[1] Y. S. Choi and R. Lui, Uniqueness of steady-state solutions for an electrochemistry model with multiple species, J. Diff. Equations 108, 424-437 (1994)

[2] Y. S. Choi and R. Lui, Global stability of solutions of an electrochemistry model with multiple species, J. Diff. Equations (to appear)

[3] Y. S. Choi and R. Lui, Multi-dimensional electrochemistry model, Arch. Rational Mech. Anal. (to appear)

[4] D. Gilbarg and N. S. Trudinger, Elliptic Partial Differential Equations of Second Order, 2nd edition, Springer-Verlag, 1983 